\title{
Roundoff-induced Coalescence of Chaotic trajectories
}

\author{
Lech Longat, Evaldo M. F. Curado, \\ International Centre of Condensed Matter Physics, Universidade de Brasília, 70919 - 970 Brasília, DF, Brazil. \\ ${ }^{\ddagger}$ Centro Brasileiro de Pesquisas Físicas, 22290-180, Rio de Janeiro, RJ, Brazil
}

(November 20, 2018)

\begin{abstract}
Numerical experiments recently discussed in the literature show that identical nonlinear chaotic systems linked by a common noise term (or signal) may synchronize after a finite time. We study the process of synchronization as function of precision of calculations. Two generic behaviors of the average coalescence time are identified: exponential or linear. In both cases no synchronization occurs if iterations are done with infinite precision.
\end{abstract}

PACS numbers: $05.45 .+\mathrm{b}, 05.40 .+\mathrm{j}$

It is well known that deterministic systems may exhibit a chaotic behavior [1]. Typically, two chaotic orbits initially displaced only slightly from each other deviate exponentially with time approaching separation on the order of the strange attractor size. In practice it means that small errors make longtime evolution of nonlinear, dynamical systems unpredictable [1]. Thus, identical chaotic systems are not expected to synchronize.

Recently, in a series of papers [2], it has been demonstrated that synchronization could nevertheless be achieved if the systems are linked by a common signal or noise term of an appropriate strength. That is, if we take a chaotic map and start numerical evolution of two arbitrarily chosen initial points subject to the same sequence of noise, the resulting trajectories will collapse after a finite number of iterations.

Two cases could be distinguished. The first one, discussed by Pecora et al. [2] and Fahy et al. [2] (PF), defines the collapse as a process where the average distance between the trajectories converges exponentially to zero. According to PF such synchronization takes place if the largest Lyapunov exponent becomes negative.

A different scenario was found by Maritan and Banavar (MB) [4. They claimed that the coalescence also may occur abruptly, without any specific time dependence of the average distance between the trajectories. As an example they studied the well-known one- dimensional logistic map [1, 5, 9] in the presence of additive noise

$$
x^{\prime}=4 x(1-x)+W \eta
$$

where $0 \leq x \leq 1 ; \eta$ is a random number chosen uniformly from the interval -1 to +1 and $W>0$ is the strength of the noise. The values of $\eta$ violating the bounds $0 \leq$ $x^{\prime} \leq 1$ were discarded and a new $\eta$ was chosen. They found that for $W>W_{c}\left(W_{c} \approx 0.55\right)$ and after about $10^{6}$ iterations the trajectories of almost any two points became identical and follow a single random trajectory. As conclusion they claimed that in the limit of infinite time and finite precision all trajectories should collapse for $W>W_{c}$. Thus at $W=W_{c}$ one would get a kind of phase transition. Several data were exhibited to confirm the collapse and the existence of the transition. 
Recently Pikovski [5] has suggested that the synchronization observed by MB is a numerical effect of finite precision of calculations. However no systematic analysis has been given. It is therefore of interest to investigate the effect of computer roundoff on the coalescence, which we would like to propose in this letter. We show that the average coalescence time (or simply the relaxation time), $T$, for the two orbits of the model (1) follows the exponential law: $T=\mathrm{e}^{A(W)+B(W) N}$ with $A$ and $B$ being functions of the noise strength; $N$ is the precision of the calculations. This law, which is different from the one suggested in [5], proves that statistically the coalescence never occurs for a map or flow of infinite precision.

We also show that no nontrivial critical value $W_{c}$ exists. Even for $W<0.5$ the collapse does occur. If we consider finite precision iterations and $W \geq W_{\min }=$ $0.5 \times 10^{-N}$ then the two different trajectories should always collapse. As conclusion one can say that the limit discussed in the paper of MB (first, time going to infinity, then the precision going to infinity) is trivially satisfied in the sense that (almost) all two points coalesce in this limit. Finally, a synchronization by common signals observed in the chaotic systems with negative Lyapunov exponents [2] yields the relaxation time linear with $N$.

Our analysis starts by considering the map studied by MB, Eq.(1]). But the approach differs from that of MB in the way the map is iterated. Namely, we perform the iterations with controlled absolute accuracy by fixing the number of digits after decimal point. More specifically, for all numbers from interval $[0,1]$ with decimal representation $x=0 . a_{1} a_{2} \cdots a_{N}$ the value of $N$ is being fixed after each iteration. Here the symbol "." represents decimal point and $a_{i}=0,1, \ldots, 9(i=1, \ldots, N)$. For $x=1$ we get automatically $x^{\prime}=0$.

Finite accuracy calculations are done with programs running under control of the MAPLE package. To use the program the precision $N$ has to be fixed. We assume $N$ to vary from 1 to 16 . Next, the noise strength $W$ must be given and the two initial numbers for the trajectories chosen randomly from the interval $[0,1]$. After these preparations are done we start two independent iterations according to the prescription (11). The process continues until both trajectories became numerically identical. The corresponding number of iterations is called coalescence time and denoted $t$. The whole procedure (with fixed $N$ and $W$ ) is repeated 100 times to get $T$.

Results of the simulations are shown as triangles in Fig.(1), where $\ln (T)$ varies with $N$ for $W=0.7$. Circles represent similar simulations but with $N$ denoting relative precision i.e. the number of digits in the mantissa as in usual floating point arithmetic. Note that for the map given by Eq.(1), the absolute- and the relative precision calculations yield statistically the same results.

Additionally, two histograms representing the probability distribution function $P(t)$ that the coalescence time is $t$, are shown as insets of Fig.(1). They are constructed 
for $N=8$ and for two different samples composed of 100 and 10000 points, respectively. Though $t$ has substantial statistical fluctuations, the difference between $\ln (T)$ calculated for both samples differs by less than $0.9 \%$. Similar result is found for $N=15$ with the averages taken over 100 and 1000 points. Hence 100 realizations of the process provide a useful estimate of $\ln (T)$.

We shall observe that $T$ increases exponentially with $N$. This has been shown in Fig.(1) by plotting a line $\ln (T) \simeq 2.297+0.765 N(16 \geq N \geq 3)$ of the best least - square fit, which on the average is good to within better than $2.5 \%$. Interestingly, similar linear dependence of $\ln (T)$ on $\mathrm{N}$ is predicted for other values of $W$. This indicates that (statistically) two trajectories never collapse in the limit of infinite precision.

At this point we would like to turn to the results of MB. They found coalescence of the trajectories for $W=0.6$ but not for $W=0.3$, in both cases the number of iterations being $5 \times 10^{5}$. Clearly, for $W=0.3$ the number of iterations they performed was much too small to see coalescence. We found that for double precision experiments and for $W=0.5-0.6$, time $T$ is orders of magnitude smaller than that for $W=0.1$ or $W=0.3$. More specifically, for $W=0.1$ the collapse is expected after about $10^{14}$ iterations while for $W=0.3$ after $10^{12}$ iterations. This effect is illustrated in Fig.(2), where $\ln (T)$ is plotted as a function of $W$ for different values of $N$. As previously averages are taken over $100 t$ 's. Again the influence of scatter of the data on $\ln (T)$ is tested for $N=3$ and $N=7$ where additionally the averages are taken over 1000 experiments. In both cases the results are practically independent of the sample size. For $N=3$ this is shown in Fig.(2) where triangles represent averages over 100 experiments and circles over 1000. It is seen that $T$ is high for small values of $W$ and decreases with increasing $W$. A minimum of $T$ is found around $W=0.6$. Then $T$ increases again to finally saturate for $W \geq 1$. This behavior is observed for all values of $\mathrm{N}$ studied and its origin will be explained later in the paper.

Now one can easily understand the results of MB, presented in their Fig.(2). A maximum of the probability that the two trajectories collapse (denoted as $\lambda$ in $(-1)$, corresponds to our minimum of $T$ (see Fig.(2)). Likewise, the decrease of $\lambda$ for $W>0.6$ is connected with our increase of $T$. The difference between our results and theirs is that we do not observe any transition around $W=0.5$. Generally we find that collapse takes place for all values of $W$ such that $W \geq W_{\min }$. This has been checked for $N \leq 8$. For higher values of $N$ time $t$ becomes too long and calculations are practically impossible.

In order to understand why $W$ must be nonzero to get collapse please note that any chaotic map looses its chaotic character when iterated with a finite accuracy [10]. Take, for example, the logistic map (11) and fix accuracy to $N=3$. The domain of the map consists in this case $10^{3}+1$ states (boxes) equal 
$(0,0.001,0.002, \cdots, 0.999,1)$. For $W=0$ these numbers evolve either to one of the fixed points: $x=0$ or $x=0.750$ or to a cycle of length $13:\{0.109,0.190,0.204$, $0.328,0.388,0.416,0.616,0.650,0.882,0.910,0.946$, $0.950,0.972\}$. Thus, in the absence of noise the trajectories belong to periodic orbits or fixed points and only accidentally they can collapse. For $W \neq 0$ the structure of the cycles is destroyed. Even the smallest numerical value of the noise $\left(W=W_{\min }\right)$, which either does not modify the iterated states or modifies them by \pm 0.001 (each realization being of the same probability equal $1 / 3$ ), is sufficient to have nonzero probability that all initial states are again accessible from any other state after a finite number of iterations. Interestingly, $W_{\text {min }}$ does not depend on detailed structure of periodic orbits indicating that any sort of noise that restores ergodicity would yield collapse of the trajectories. The same statements hold for arbitrary $N$. Instead, if we consider infinite accuracy the probability that two numbers coming out of iterations are different is one. As we are in chaotic region, where dynamics is strongly sensitive to the initial conditions, these two points will move away from each other in the forward iterations.

Another new feature of the logistic map found in our simulations and displayed in Fig.(2) is behavior of the curves $\ln (T)$ as function of $N$. Namely they all scale linearly with $N$, i.e. $\ln (T)=A(W)+B(W) N$, with $W$ dependent coefficients. Using the data shown in Fig.(2) we estimated $A(W)$ and $B(W)$ by performing linear least square fit for each $W$ separately. The obtained set of points is given in Fig.(3). These data show that for $W \approx$ 1 the time $T$ behaves as $10^{N / 2}$ and for $W=0.6$ as $10^{N / 3}$, which differs from the $10^{2 N}$-law suggested in [5].

Finally, the Fig.(3) allows one to estimate $T$ for $W=$ 0.3 and for $N=15$ as being of order of $10^{12}$. This is the reason why in experiments of $\mathrm{MB}$, which dealt with $10^{6}$ iterations, the collapse for $W=0.3$ statistically has not been observed.

A further insight into the coalescence process and its connection to the accuracy could be achieved by converting the logistic map (1) into the 2y modulo 1 map [7]

$$
y^{\prime}=2 y \quad \text { modulo } 1+g(W, \eta, \ldots) .
$$

This is done in practise by a change of variables: $x=\frac{1}{2}[$ $1-\cos (2 \pi y)]$. Note that the transformed noise, denoted by $g(W, \eta, \ldots)$, depends at time $n$ on the system states prior to $\mathrm{n}$. This dependence, represented by dots in Eq.(2), appears quite nontrivial and will not be of our concern here. Instead we will discuss a simple case where precise form of $g(W, \eta, \ldots)$ is irrelevant. Again the values of $g$ violating the bonds $0 \leq y^{\prime} \leq 1$ are discarded.

Suppose that we study the binary version of the map (2) in the interval $[0,1)$. That is we iterate the map by representing each number (including the noise term) as a binary decimal $0 . a_{1} a_{2} a_{3} \cdots a_{N} \cdots \equiv \sum_{i=1}^{\infty} 2^{-i} a_{i}$, where 
each of the digits $a_{i}$ is either 0 or 1 . With this simple change of base the iterations of the map (2) could be viewed as moving the decimal point "." one position to the right (Bernoulli shift).

A binary number of accuracy $N$ could now be introduced as the one for which $a_{j}=0, \forall j>N$. Consider two arbitrary numbers $x$ and $y$ of binary accuracy $N$ and assume that their binary representations are of the form $0 . x_{1} x_{2} x_{3} \cdots x_{N}$ and $0 . y_{1} y_{2} y_{3} \cdots y_{N}$, respectively. The first iteration moves the points one step to the right yielding $x^{\prime}=0 \cdot x_{2} x_{3} \cdots x_{N} 0$ and $y^{\prime}=0 \cdot y_{2} y_{3} \cdots y_{N} 0$. As the last digit (zero) is now the same for both numbers $x^{\prime}$ and $y^{\prime}$ the noise converts them into two numbers with the same digit (the $N$-th digit of the noise) at position $N$ after the point. Successive iterations in the presence of noise increase the number of identical digits by one making the coalescence time $t \lesssim N$ and, consequently, $T$ must be a linear function of $N$. Similar linear behaviour of $T$ with $N$ is predicted for the PF models [2]. This follows directly from the fact that the average distance, $d$, between the PF trajectories at the time $T$ is $d=A_{0} 10^{-T / \lambda}$, where $A_{0}$ and $\lambda$ are model dependent parameters. Comparing $d$ with $10^{-N}$ yields $T \sim \lambda N$.

Let us now come back to the logistic map and comment two issues: (a) why the minimal $T$ is found for $W \gtrsim 0.5$ and (b) how $T$ could be connected with the properties of the map and of the noise. An important point to note (see also (4) is that, in general, chaotic maps consist of iterations that are composed of two parts, the one which stretches the distance between the points and the other where the distance is enlarged. Consider the distance $d_{l}$ between images of $x$ and $y$

$$
d_{l}: \mathcal{S} \ni(x, y) \rightarrow 4|x-y||1-(x+y)|,
$$

where $\mathcal{S}=[0,1] \times[0,1]$. Then the distance contracting region, $\Omega$, is given by the condition: $3 / 4<x+y<5 / 4$, where $(x, y) \in \mathcal{S}$. That is, $\forall(x, y) \in \Omega$ : $\quad d_{l}(x, y)<$ $|x-y|$. If we require that the reduction of the distance is smaller than $\varepsilon$ i.e. $d_{l}(x, y)<\varepsilon|x-y|$ then the states $(x, y)$ must be restricted to a strip $\Omega_{\varepsilon} \subset \Omega$ given by the inequality $1-\varepsilon / 4<x+y<1+\varepsilon / 4$. The area $\tilde{\Omega}_{\varepsilon} \subset \Omega_{\varepsilon}$, where shrinking of the distance is being the strongest, is found around $(x, y)=(1 / 2,1 / 2)$, where $d_{l}\left(1 / 2 \pm \varepsilon / 2,1 / 2 \pm \varepsilon^{\prime} / 2\right)=\left|\varepsilon^{2}-\left(\varepsilon^{\prime}\right)^{2}\right|$.

To proceed further we assume that the random orbits are described by the joint probability distribution function $\rho_{W}(x, y)((x, y) \in \mathcal{S})$ and apply the noise to all pairs of numbers $(x, y)$. Now the probability $P_{W}(\varepsilon)$ that this procedure yields two numbers such that after applying the map, they are moved into the contracting area $\Omega_{\varepsilon}$ is given by the formula

$$
P_{W}(\varepsilon)=\int_{0}^{1} \int_{0}^{1} d x d y \rho_{W}(x, y) \frac{\left|\mathcal{I}(x, y ; W) \cap \Omega_{\varepsilon}\right|}{|\mathcal{I}(x, y ; W) \cap \mathcal{S}|}
$$

where $\mathcal{I}(x, y ; W)$ is the interval from $(x-W, y-W)$ to $(x+W, y+W)$ of length $|\mathcal{I}(x, y ; W)|=2 \sqrt{2} W$. The key 
quantity entering the formula (4) is $\rho_{W}(x, y)$. Clearly, $\rho_{W}(x, y)$ should be peaked about the diagonal $x=y$ with strong (absolute) maximum at $(x, y)=(1,1)$. This observation follows from the fact that the logistic map exhibits singular density of states near 1 and 0 and that the points around 0 are images of those around 1 . As after each iteration the noise is added (with the constraint that the pair of numbers stays within the basin of attraction of the map) the points around 0 are obtained less frequently. Thus, to get maximal probability $P(\varepsilon)$ the integration in (何 should include the region around $(1,1)$ which implies that $W \gtrsim 0.5$. On the other hand the second term under integral, which gives the probability of finding the noise-shifted point in $\Omega_{\varepsilon}$, is for $W \gtrsim 0.5 \mathrm{a}$ decreasing function of $W$ and saturates for $W>1$. Combining these two facts we conclude that $P_{W}(\varepsilon)$ should be, for $W \lesssim 1$, a decreasing function of $W$, approaching saturation for $W>1$. The saturation could be proceeded by a maximum, laying between $0.5 \lesssim W \lesssim 1$. In this case one gets minimum of $T$ for $0.5 \lesssim W \lesssim 1$. Finally, $\lim _{\varepsilon \rightarrow 0} P_{W}(\varepsilon) \rightarrow 0$ implies that $T \rightarrow \infty$ for $N \rightarrow \infty$.

The upper limit of $T$ for $W \gtrsim 0.5$ also could be found from the formula (4). As the distribution $\rho_{W}(x, y)$ is strongly peaked around $(1,1)$ the function $P_{W}(\varepsilon)$ actually gives a probability of finding a point in the area $\tilde{\Omega}_{\varepsilon}$. From (4) it follows that the frequency of getting a point in $\tilde{\Omega}_{\varepsilon}$ is proportional to $\varepsilon$ which gives $T \sim 1 / \varepsilon$. For $\varepsilon=10^{-N / 2}$ the image of any point from $\tilde{\Omega}_{\varepsilon}$ yields two numbers that are $10^{-N}$ apart indicating that the two trajectories just collapsed. Hence $T \sim 10^{N / 2}$, which is what we find for $W \approx 1$.

These observations have important practical consequences. They suggest that the coalescence time to a fixed precision could be controlled by the noise. For the map (11) this is achieved by accepting only these $\eta$ 's in (1) which give points $\left(x^{\prime}, y^{\prime}\right)$ restricted to $\Omega_{\varepsilon}$. Allowed values of $\varepsilon$ as functions of assumed $N$ and $T$ and for $W \geq 0.5$ could again be inferred from Eq.(勾).

In conclusion, we showed that in order to understand the recently observed phenomenon of coalescence of the trajectories [2, [4] the precision of the calculations must be taken into account. For all systems studied in the literature so far, the average coalescence time $T$ is either linear or exponential function of precision of the calculations. Thus, statistically, coalescence never occurs when precision is infinite. Clearly the arguments as given are of general validity and the logistic map or the tent map could serve as examples.

One may wonder as whether predicted linear (or exponential) behaviour of $T$ with $N$ could be correlated with the sign of the maximal Lyapunov exponent. If the identical chaotic systems are subject to an external noise, which is generated at each time step independently of the previous values and of the states of the systems, than the analysis proposed by Pikovsky applies [3]. In this case the largest Lyapunov exponent can be calculated from a 
single system. If this exponent is negative than, at least, for the systems studied in [3] this would imply the linear dependence of $T$ with $N$ (i.e. synchronization). However, such linear dependence does not necessary mean that the maximal Lyapunov exponent must be negative. For example, in the case of the binary tent map, where $T$ varies linearly with $N(\forall g)$, the sign of the Lyapunov exponent (for $N \rightarrow \infty$ ) could be arbitrary. Also we would like to add that the technique [3] does not apply when the noise depends on the states of the systems, Eqs.(1, 2). In this case the difference between ensembles with positive and negative Lyapunov exponents is highly nontrival [11. This issue is currently under investigations and will be reported elsewhere.

Finally, let us note that $T$ is governed by the properties of the invariant density of the chaotic map, the structure of the distance stretching area(s), and the way in which the noise is introduced. This is demonstrated for the random logistic map (1), where $T$ varies exponentially with $N$. The law we found is different from a simple $\left(10^{-N}\right)^{-2}$ dependence suggested in [5] $\left(\equiv \varepsilon^{-D}\right.$, where $D \equiv 2$ is the topological dimension of the space).

\section{ACKNOWLEDGMENTS}

This work was supported by the Conselho Nacional de Desenvolvimento Científico e Tecnológico (CNPq) and by the Financiadora de Estudos e Projetos (FINEP) in Brasil.

* Permanent Address: Department of Statistical Physics, Jagiellonian University, Reymonta 4, Kraków, Poland.

[1] See e.g. (a) H. G. Schuster, Deterministic Chaos: An introduction, VCH Verlagsgesellschaft, Germany, 1989; (b) J. Ford in "Directions in Chaos", Ed. Hao Bai-Lim, World Scientific Series On directions in Condensed Matter Physics- vol.3 (1989)

[2] (a)L. M. Pecora and T. L. Carroll, Phys. Rev. Lett. 64, 821 (1990); (b)S. Fahy and D.R. Hamann, Phys. Rev. Lett. 69, 761 (1992).

[3] A. S. Pikovsky, Phys. Letters A 165, 33 (1992).

[4] A. Maritan and J.R. Banavar, Phys. Rev. Lett. 72, 1451 (1994); Phys. Rev. Lett. 73, 2932 (1994).

[5] A. S. Pikovsky, Phys. Rev. Lett. 73, 2931 (1994).

[6] (a)M. J. Feigenbaum, Los Alamos Science 1, 4 (1980); (b) P. Coullet, J. Tresser, J. Phys. (Paris) Coll. 39, C525 (1978); (c)R. M.May, Nature 261, 459 (1976).

[7] E. Ott, Chaos in dynamical systems, Cambridge University Press 1993.

[8] C. Tsallis, A. M. C. de Souza and E. M. F. Curado, Chaos, Solitons and Fractals 6, 561, (1995).

[9] F. A. Oliveira, Phys. Rev. B 52, 1009 (1995). 
[10] C. Grebogi, E. Ott, and J. A. Yorke, Phys. Rev. A 38, 3688 (1988).

[11] G. Paladin, M. Serva, A. Vulpiani, Phys. Rev. Lett. 74, 66 (1995).

FIG. 1. Logarithm of average relaxation time versus accuracy for $W=0.7$. The straight line represents best least-square fit obeying $3 \leq N \leq 16$. Triangles represent absolute accuracy calculations while circles relative ones. For each accuracy average is performed over 100 samples. Insets show the histograms of the coalescence times for $N=8$ and for two sample sizes equal 100 and 10000, respectively.

FIG. 2. Logarithm of the average relaxation time versus the strength $W$ of the noise. Accuracies considered are $N=3$ (triangles and circles), 4 (losangles), 5 (pentagons) and 6 (hexagons). Averages are performed over 100 samples except for $N=3$ (circles) which corresponds to 1000 samples. The lines are introduced to guide eyes.

FIG. 3. Coefficients $A(W)$ (thin line) and $B(W)$ (thick line) of $\mathrm{T}(\mathrm{N})$ for the model (1). The lines are guide-to-eyes. 


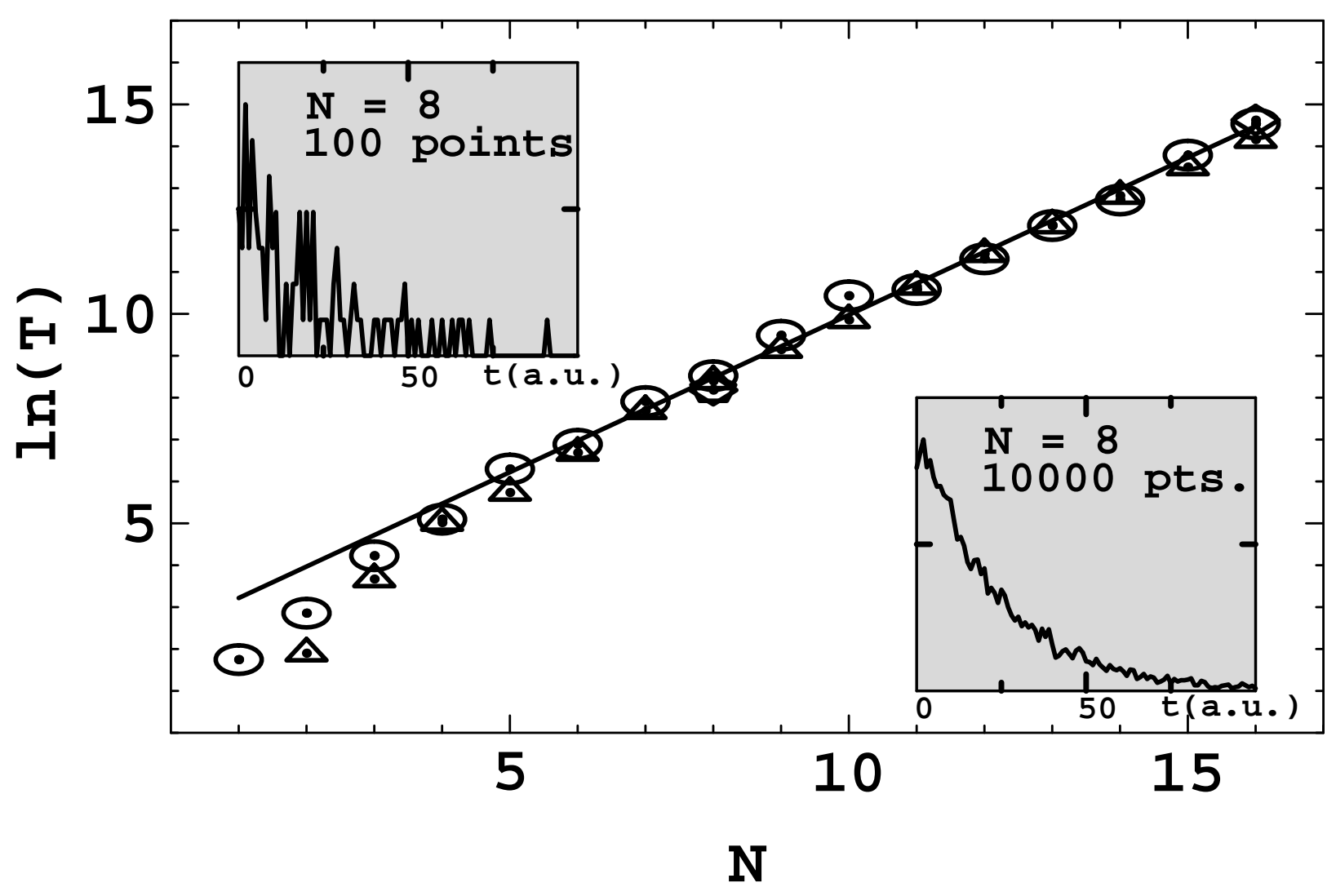




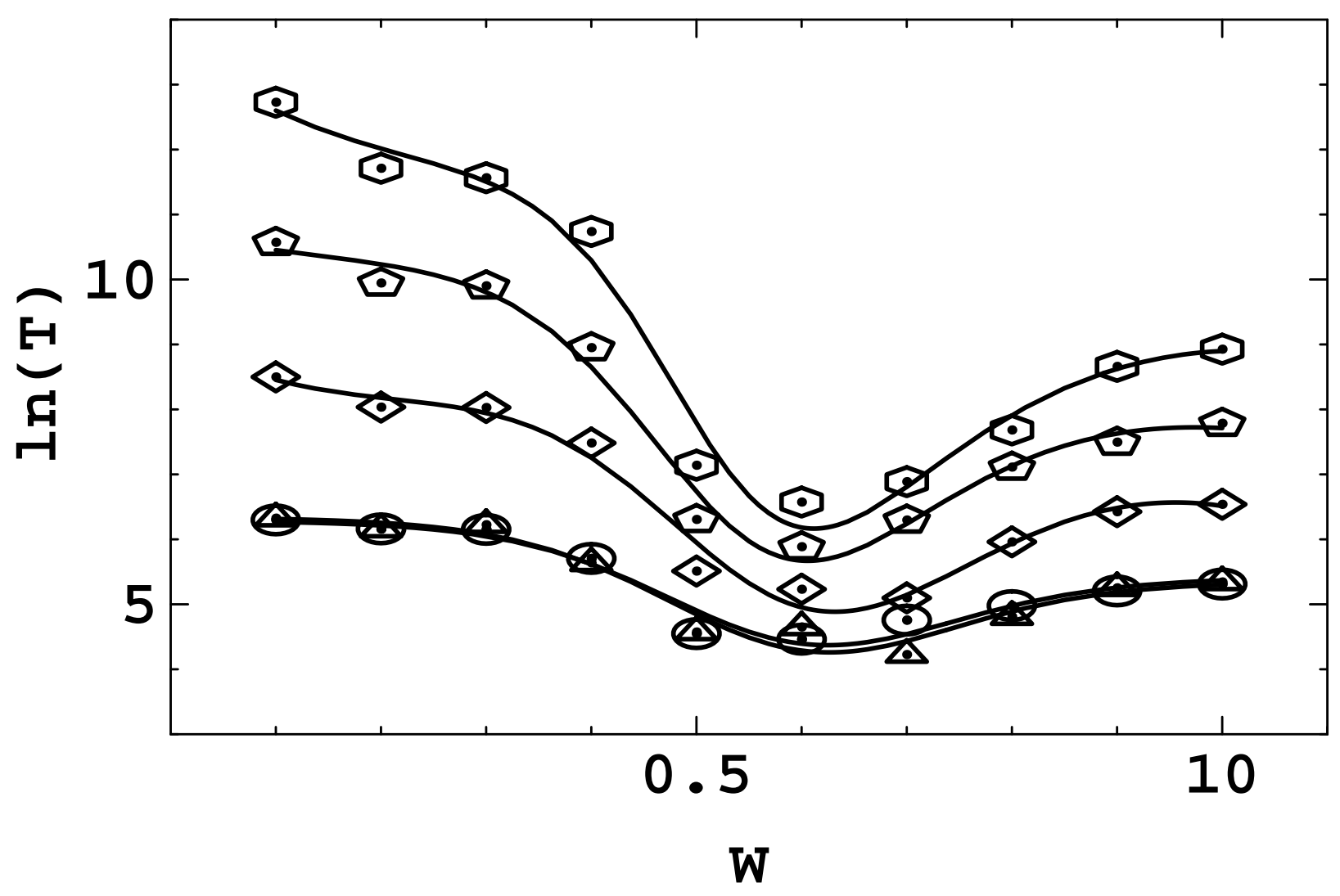




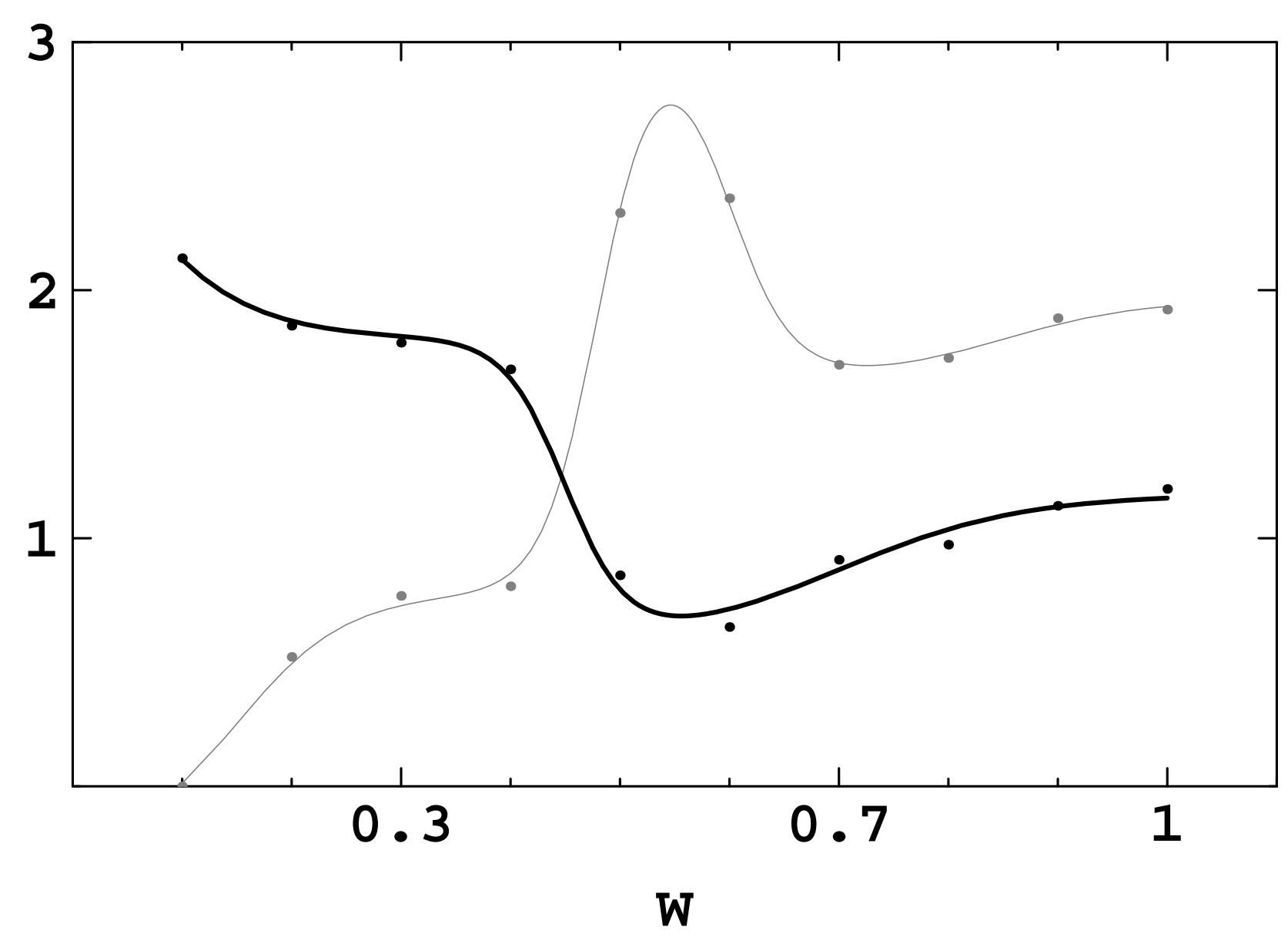

